\title{
ALGUNAS CONSIDERACIONES ÉTICAS SOBRE LA APLICACIÓN DE LA INTELIGENCIA ARTIFICIAL EN EL DEPORTE
}

\author{
Alberto Carrio Sampedro \\ Universitat Pompeu Fabra \\ alberto.carrio@upf.edu
}

Fecha de recepción: septiembre 2020

Fecha de aceptación: noviembre 2020

http://doi.org/10.15366/citius2020.13.2.004

\section{Resumen:}

La inteligencia artificial (IA) ha irrumpido recientemente en el deporte, pero sus aplicaciones ocupan ya un lugar destacado en la práctica deportiva e irán sin en aumento en los próximos años. Son muchos los beneficios que estos sistemas de tecnología avanzada pueden proporcionar al deporte. Pero las aplicaciones de la IA en el deporte no están exentas de riesgos. Entre ellos, la manipulación de los deportistas y de la competición son probablemente los más acuciantes. Pero hay otros, como la modificación radical de la gobernanza del deporte y las competiciones deportivas que quedarán completamente mediatizadas por esta tecnología disruptiva. En este artículo propongo un marco jurídico y ético que nos permita disponer de un uso fiable de la IA y respetuoso con los derechos humanos de los deportistas y los compromisos éticos del Movimiento Olímpico.

Palabras clave: inteligencia artificial, deporte, Movimiento Olímpico

Title: THE ETHICS OF AI AND ADVANCED TECHNOLOGY IN SPORTS

\begin{abstract}
:
Artificial intelligence (AI) has recently burst into sports, but it already plays an important role in and will continue to do so in the coming years. There are many benefits that such advanced technology can provide to sport. However, AI applications in sports are not without risk. Among them, the manipulation of athletes and the competition are probably the most pressing. There are also others, such as the radical modification of sport governance and sport competitions that will be completely mediated by this disruptive technology. In this article, I propose a legal and ethical framework that enables a reliable use of AI and consistent with the human rights of athletes and the ethical commitments of the Olympic Movement.
\end{abstract}

Keywords: artificial intelligence, sport, Olympic Movement

\section{Introducción.}

Los sistemas de inteligencia artificial (IA) y la ingeniería tecnológica avanzada (advanced tech engineering) constituyen probablemente, junto con la edición genética, la expresión más acabada de la antigua aspiración humana de jugar a ser Dios. Por primera vez en la historia de la humanidad somos capaces de crear sistemas autónomos que, imitando el aprendizaje humano realizan tareas complejas de forma mucho más eficiente que los propios seres humanos. Los sistemas de IA calculan y predicen situaciones, aprenden de sus propios errores, o por ser más exactos de los errores padecidos en la programación, y adaptan constantemente su comportamiento a situaciones cambiantes hasta el punto de ser absolutamente independientes del control humano.

No cabe duda de que la IA representa un gran avance tecnológico que puede contribuir a mejorar las condiciones de vida de las personas. Los sistemas de IA facilitan la adopción de decisiones complejas, mejoran la atención sanitaria personalizada y proporcionan información 
relevante que permite una distribución más eficiente y equitativa de los recursos y pueden tener gran relevancia a la hora de combatir la crisis climática que padecemos. Las aplicaciones de los sistemas de IA en el deporte son también notables e irán en aumento en los próximos años. Por de pronto han demostrado ser eficaces en la prevención de lesiones, asistencia al arbitraje, en la preparación física de los deportistas y en la mejora de las estrategias competitivas. La IA incrementa la seguridad de los estadios y es empleada para involucrar a los aficionados en el ámbito del deporte y en las campañas que promueven la integridad deportiva.

Ahora bien, los sistemas de IA pueden tener también graves efectos en el mundo del deporte. Pueden, ciertamente, afectar negativamente a los derechos básicos de los deportistas y resto de personas implicadas en el deporte. No solo porque puedan poner en riesgo valores tan preciados como la autonomía y dignidad individual de los deportistas, sino también porque pueden ser nocivos para la salud y restringir injustamente las posibilidades de éxito deportivo. En definitiva, la IA puede contribuir a aumentar la desigualdad y modificar profundamente la concepción que tenemos del deporte y, en particular, de la competición como ejemplo de mérito individual.

En este artículo trataré de analizar y explicar brevemente los impactos positivos y negativos de la IA en el deporte. Pero para ello será necesario adentrarnos siquiera brevemente en el concepto y diferentes aplicaciones de la IA.

El artículo se desarrollará como sigue. En la siguiente sección haré una breve introducción a los usos y aplicaciones actuales de la IA. A continuación, definiré, muy tentativamente, qué se entiende por IA y describiré algunas de las preocupaciones actuales de las aplicaciones cotidianas de la IA. La sección 3 está dedicada a explicar y analizar algunos de los usos actuales y futuros de la IA en el deporte. Esta perspectiva descriptiva cambia en la sección 4, en la que me ocuparé de algunas cuestiones normativas relativas a los desafíos que plantea la IA a los Derechos Humanos. Este enfoque normativo se mantiene en la sección 5, en la que propondré un conjunto de principios éticos que permitan encauzar los usos actuales y el futuro desarrollo y despliegue de la IA en el deporte. Para finalizar trataré de presentar algunas recomendaciones sobre las aplicaciones de la IA al deporte.

\section{2. ¿Qué es la Inteligencia Artificial?}

Nuestra vida cotidiana se ha visto envuelta desde hace algún tiempo por diversos usos y aplicaciones de los sistemas de IA. Siri y Alexa son probablemente las aplicaciones más conocidas. Pero la ingeniería en sistemas de IA está también detrás de muchas acciones diarias. Las transacciones seguras que hacemos en nuestras cuentas bancarias desde aplicativos móviles, la atención telefónica personalizada a través de terminales automáticos o el creciente aumento de robots utilizados para el cuidado de niños y ancianos, la temperatura y consumo energético de nuestras viviendas o la atención robotizada en hostelería. La IA se nutre de los algoritmos que nos recomiendan un buen restaurante, un lugar donde pasar nuestras vacaciones, o los que se emplean en los sistemas de salud públicos y privados para hacer más eficientes las terapias e intervenciones quirúrgicas. La mejora en los sistemas de reconocimiento facial no solo permite a la policía identificar y aislar individuos en una manifestación o ser utilizado como forma de control de acceso en los aeropuertos. En esta época de pandemia del COVID-19 el uso de los sistemas inteligentes ha ido en aumento en los edificios públicos y privados y en el control de los exámenes que realizan los estudiantes en las universidades. La IA se emplea en definitiva para mejorar la seguridad individual al mismo tiempo que nos sitúa ante el riesgo de una nueva carrera armamentística por la posesión y control de las denominadas armas letales autónomas (LAW por sus siglas en inglés)

Como ocurre en cualquier otro ámbito de la vida, la IA puede ser utilizada de diferentes maneras en el deporte y algunas de ellas no son seguras, ni justas ni éticas. La IA es una herramienta eficaz en la lucha contra el dopaje al mismo tiempo que alumbra nuevas formas de alteración de la competición como el ciberdopaje y neurodopaje. La IA ha demostrado ser útil 
para mejorar la resistencia y el rendimiento deportivos, pero también ha puesto de manifiesto nuevos riesgos para la salud y el creciente uso de los sistemas de detección y seguimiento amenazan seriamente la autonomía y privacidad de los deportistas.

Así las cosas, parece que, pese a su novedad, la IA queda sujeta a aquella vieja máxima de Campoamor, dado que su bondad o maldad depende, si no del cristal con que se mira, de los usos que se hagan de ella. Y dado que el deporte ha sido desde siempre un ámbito proclive a la experimentación de los avances científicos y tecnológicos más vale prevenir que lamentar las consecuencias indeseables que pueden tener. Particularmente importante es evitar la repetición de episodios bien conocidos de la historia del deporte en los que la salud e integridad física de los deportistas ha sido sacrificada en aras de causas destinadas a engordar el ego individual de los investigadores, las arcas de clubes y patrocinadores o como instrumento de propaganda política.

Ahora bien, lo realmente novedoso del desafío que plantea la IA radica en la creciente autonomía de estos sistemas, o lo que es lo mismo en el grado de opacidad de su funcionamiento para los seres humanos. Piénsese por ejemplo en lo que ocurre con las denominadas redes generativas antagónicas (GAN por sus siglas inglesas) que se le alimentan a partir de lo que se conoce como «adversarios generativos». Es decir, sistemas de aprendizaje basado en dos redes neuronales que se retroalimentan en una especie de competición continua de suma cero que mejora constantemente el conocimiento e interacción de estos sistemas como el medio. Un buen ejemplo de este desafío lo encontramos en el creciente perfeccionamiento de los denominados deportes electrónicos (e-sports) y los sistemas de aprendizaje autónomo avanzado. Recuérdese el bien conocido caso Deep Blue, el primer sistema computacional capaz de derrotar a un Gran Maestro de Ajedrez como Kasparov en 1997. Deep Blue tenía una clasificación Elo por encima de 2700 (Kasparov había alcanzado 2851 en 1999). Hace escasamente 3 años que el sistema AlphaZero fue lanzado por DeepMind cuya clasificación Elo se estima en 3600. Pero lo más sorprendente es que AlphaZero puede llegar a ser imbatible a partir de una programación básica de las reglas constitutivas del ajedrez y de hacerlo, además, en cuestión de horas. El problema que pone ante nuestros ojos la asombrosa capacidad de este sistema es que los procesos que utiliza para mejorar su comportamiento quedan fuera del conocimiento humano. Es decir, una vez que comienza el proceso de aprendizaje se ignora los pasos que realiza para llegar a ser imbatible a partir de los algoritmos iniciales de programación.

En lo que sigue me centraré en aquellos usos de lo que podemos considerar IA en sentido débil. Es decir, aquellos usos de la IA como el reconocimiento facial, los algoritmos empleados en la atención médica o aquellos que sirven para mejorar el entrenamiento y evitar lesiones a los deportistas, o para cuestiones aparentemente más lúdicas como jugar al ajedrez o al fútbol con máquinas. En otras palabras, los usos de la IA para tareas específicas destinadas a interactuar con los humanos. La IA en sentido fuerte, o lo que es lo mismo, los sistemas de IA que superan la eficiencia y capacidad de la cognitividad humana son, sin embargo, los más preocupantes dado que queda fuera de nuestro control. Precisamente porque estos sistemas están ya operativos en el mundo del deporte es preciso prestar la debida atención y disponer de unas bases éticas y jurídicas adecuadas para prevenir los efectos que puedan tener.

\subsection{Una definición tentativa de IA}

Los avances que se han producido en la IA desde sus inicios a mediados del siglo pasado $\mathrm{y}$, en particular, durante el último tercio del siglo XX y lo que llevamos del presente, han obligado a adaptar constantemente el concepto de IA. El sinfín de dispositivos y aplicaciones que ha generado la constante evolución de la ingeniería en tecnología avanzada han ido incorporándose a la definición de IA. Bien podría decirse que el término IA da cobijo a una diversidad de sistemas que tienen en común la capacidad de interactuar autónomamente con el medio en el que se encuentran. Un buen ejemplo de esta concepción de la IA como término paraguas lo constituye la siguiente definición de la Comisión Europea: 
«La inteligencia artificial (IA) se refiere a los sistemas que muestran un comportamiento inteligente analizando su entorno y tomando medidas — con cierto grado de autonomía — para lograr objetivos específicos.

Los sistemas basados en la IA pueden estar basados puramente en software, actuando en el mundo virtual (por ejemplo, asistentes de voz, software de análisis de imágenes, motores de búsqueda, sistemas de reconocimiento de voz y de rostros) o la IA puede estar incorporada en dispositivos de hardware (por ejemplo, robots avanzados, automóviles autónomos, aviones no tripulados o aplicaciones de Internet de las cosas).» ${ }^{1}$

El problema que presenta una definición como esta es que, al igual que ocurre con todos los términos paraguas, se presta a interpretaciones ambiguas que pueden generar confusiones importantes. De ahí que sea conveniente diferenciar entre el concepto de IA y las diferentes aplicaciones de la misma a las que ha dado lugar la ingeniería tecnológica. Por otra parte, es necesario distinguir asimismo entre los sistemas de AI en atención a las funcionalidades básicas que presentan. Es decir, entre los sistemas de aprendizaje automatizado, como las aplicaciones informáticas, los sistemas de procesamiento y análisis de datos; de los sistemas que además emplean este aprendizaje para incrementar constantemente su autonomía e independencia de los seres humanos. ${ }^{2}$ La eficiencia que muestran los primeros combinada con la creciente autonomía de los segundos permite hacernos una idea de la dimensión del problema al que nos enfrentamos.

Las aplicaciones de la IA se han visto incrementadas a partir de los avances experimentados en la denominada Mecatrónica, un ámbito multidisciplinar que combina varios tipos de ingeniería, en particular la robótica y la ingeniería electromecánica. El crecimiento de la industria Mecatrónica proporciona ya una amplia gama de productos de robótica y sistemas de alta tecnología, como pueden ser los robots industriales de producción masiva, los robots utilizados para envasar y empaquetar productos, los aviones militares no tripulados o los automóviles inteligentes. Este tipo de ingeniería es el que se encuentra detrás de Robocup ${ }^{\circ}$, los famosos robots futbolistas cuyos creadores han prometido que a mediados del presente siglo podrían enfrentarse con éxito a un combinado internacional de los mejores jugadores de fútbol mundial. El reto en concreto que lanza Robocup® es el siguiente: un equipo de robots humanoides será capaz de enfrentarse y ganar en un partido de fútbol al último vencedor de la Copa Mundial del Fútbol organizada por FIFA. ${ }^{3}$

Ahora bien, sea cual sea uso, el desafío que presentan los sistemas inteligentes es que cuanto mayor es su autonomía, la capacidad de realizar tareas de forma independiente, menor es el control que tenemos sobre ellos. Así las cosas, si tenemos en cuenta la capacidad ilimitada de adaptación que tiene un sistema de reforzamiento continuo del aprendizaje y los constantes avances en robótica no es difícil imaginar un futuro en el que los robots inteligentes queden fueran de nuestro control. Es decir, en una transformación tan radical de nuestra vida que hasta hace pocos años tan sólo podíamos concebir como ciencia ficción, pero que hoy es ya una realidad. Así lo demuestra, nuestra creciente interacción con los sistemas de IA. Buen ejemplo de ello son los denominados Digital Twins, la réplica digital de entidades físicas vivas o no y los Cyborgs, la integración de sistemas de IA dentro del cuerpo humano. Todo ello nos coloca en una tesitura en la que prescindir de la valiosa ayuda que pueden prestarnos estos sistemas sería tanto como renunciar a un futuro más prometedor y fiar completamente nuestras decisiones a ellas puede tener consecuencias incalculables.

\footnotetext{
${ }^{1}$ Communication from the Commission to the European Parliament, the European Council, the Council, the European Economic and Social Committee and the Committee of the Regions on Artificial Intelligence for Europe, Brussels, 25.4.2018 COM(2018) 237 final

${ }^{2}$ Véase European Group on Ethics in Science and New Technologies (2018). Statment on Artificial Intelligence, Robotics and Autonomous systems, p. 6. Available at https://ec.europa.eu/research/ege/pdf/ege_ai_statement_2018.pdf

${ }^{3}$ Los objetivos de Robocup ${ }^{\circledR}$ pueden encontrarse en su sitio web: https://www.robocup.org/objective
} 
En resumen, al mismo tiempo que la IA tiene muchas aplicaciones positivas, como la accesibilidad a un sinfín de datos, la posibilidad de realizar contratos inteligentes, monedas encriptadas, mejorar el rendimiento deportivo o evitar lesiones, todas ellas cada vez más presentes en nuestra vida cotidiana; los sistemas inteligentes más avanzados nos sitúan ante el riesgo de perder el control de estos sistemas y con ello de nuestra propia autonomía.

\section{Los sistemas de IA aplicados al deporte.}

La IA tiene muchas aplicaciones en el deporte. No es por tanto de extrañar que su uso vaya constantemente en aumento. Probablemente una de las acciones más sorprendentes de la utilización de sistemas de IA en el deporte, concretamente del manejo del Big data, fue la decisión tomada por Louis Van Gaal en los cuartos de final del Campeonato Mundial de Fútbol de la FIFA en 2014. Como se recordará, justo antes de la tanda de penaltis que decidiría el partido, Louis Van Gaal cambió al portero titular, Jasper Cillessen, por Tim Krul que era suplente. La decisión vino motivada en parte por la mayor envergadura de Krul, que tiene una estatura superior en $6 \mathrm{~cm}$ a la de Cillessen, pero también por una cuestión estratégica que tenía que ver con el tiempo del que disponían los asesores de los lanzadores de penaltis de Costa Rica para examinar las estadísticas de detención de penaltis por ambos porteros. Es decir, la estrategia de Louis Van Gaal consistió no solo en disponer de un portero de mayor envergadura sino también sorprender al rival y obligarlo a lanzar los penaltis sin la ayuda de una información muy valiosa a la que acuden todos los asesores de los lanzadores desde el momento en que el Big Data, hizo aparición en el deporte. Como es sobradamente conocido Krul detuvo dos de los cinco penaltis y con ello la selección holandesa se clasificó para semifinales.

La asistencia arbitral a través de imágenes grabadas es otra de las aplicaciones tecnológicas que desde hace varias años se aplican al deporte y que últimamente ha popularizado la introducción del conocido VAR en el fútbol profesional por parte de la FIFA. Pero el empeño innovador en el fútbol profesional no termina aquí. En realidad el VAR parece ser solo el comienzo del uso de la tecnología en la asistencia arbitral dado que, como la propia FIFA ha anunciado recientemente, se está estudiando la posibilidad de sustituir a los jueces de línea por robots equipados con software inteligente que evitaran los errores humanos a la hora de señalar las posiciones adelantadas de los delanteros en los pases de ataque, los conocidos fuera de juego y otros incidencias del juego. Así las cosas, la sustitución completa del arbitraje humano podría ocurrir en un futuro muy lejano. De acuerdo con las previsiones que maneja la FIFA la eliminación del VAR y la completa sustitución de los equipos arbitrales por sistemas de inteligencia artificial podría tener lugar en un periodo de entre 5 y 10 años. Pero aparte del Big Data y la asistencia arbitral en un sinfín de deportes, los sistemas de IA tienen muchas otras aplicaciones en el deporte. Veamos muy brevemente algunas de ellas:

\subsection{Mejora de los entrenamientos.}

El uso de los sistemas de IA para mejorar el entrenamiento de los deportistas no solo va en aumento, sino que ha demostrado tener resultados altamente positivos, como ahora veremos con algunos ejemplos.

La incorporación de los sistemas de IA proporciona a los deportistas no pocas ventajas derivadas del aprendizaje automático de estos sistemas que permite agrupar y clasificar los datos de forma eficiente y mejorar el rendimiento individual y colectivo de los deportistas. Estos sistemas son empleados para evaluar secuencias de movimientos, la resistencia y el rendimiento individuales en función de la superficie, de la velocidad del juego o de circunstancias cambiantes en función del tipo de competición. Lo cierto es que la utilización de estos sistemas es hoy una realidad en las competiciones deportivas y su uso va en aumento incluso en el deporte recreacional. ${ }^{4}$ Un buen ejemplo del empleo de estos sistemas de IA y la tecnología asociada a ellos lo ofrecen los recientes Juegos Olímpicos de Verano de Río de Janeiro de 2016, que

\footnotetext{
${ }^{4}$ Baca (2012), McCullagh (2010) Novatchkov (2014)
} 
probablemente fueron el primer gran evento deportivo internacional en el que el uso generalizado de estos sistemas demostró su eficacia. Así lo reconoció al menos la campeona olímpica de boxeo en la categoría de peso mosca, Nicola Adams, quien afirmó que cuando accedía al ring conocía perfectamente qué tácticas debía utilizar en cada momento para ser eficiente en cada asalto y poder ganar los combates. Algo similar ocurrió con Philiph Buhl, el regatista olímpico alemán, quien utilizó un sistema de IA para poder predecir con precisión la corriente en cada tramo de la Bahía de Guanabara de Río de Janeiro en la que se disputaba la competición. Así lo demuestra, en fin, la preparación llevada a cabo por el equipo de boxeo del Reino Unido que se benefició de la asistencia proporcionada por el software desarrollado por la Universidad de Sheffield Hallam.

\subsection{Coaching asistido.}

La eficiencia en la gestión de datos de los sistemas de IA es también de mucha utilidad a la hora de desarrollar y mejorar las estrategias competitivas durante los entrenamientos y en el transcurso de la competición. La utilización de estos sistemas permite conocer a los entrenadores y resto del equipo técnico el rendimiento individual y colectivo en tiempo real, predecir las posibilidades de evolución de la competición, y valerse de las recomendaciones de estos sistemas a la hora de sustituir jugadores, modificar las estrategias y un largo etcétera de aplicaciones de mucha utilidad dentro y fuera de la competición. No en vano estos sistemas son actualmente empleados en deportes de equipo como el baloncesto, rugby, fútbol y ciclismo.

\subsection{Control de dopaje.}

La combinación de la tecnología y los sistemas de IA han demostrado su eficiencia en la prevención y lucha contra el dopaje. Como es sabido la Agencia Mundial Antidopaje (AMA) ha sido desde sus inicios pionera en la implementación de nuevas formas de control y lucha contra el dopaje tanto por sus consecuencias en la salud de los deportistas cuanto por su capacidad de alterar la competición. Precisamente el interés de la AMA en hacer uso de esta tecnología para la geolocalización de los deportistas fuera de la competición mediante el implante de un chip en el cuerpo de los deportistas despertó un gran interés y un amplio debate debido precisamente a las cuestiones éticas que presenta el uso de este tipo de tecnología avanzada. ${ }^{5}$ Las razones para rechazar el implante de estos dispositivos son, de acuerdo con la Comisión de ética de la AMA de dos tipos, éticas e instrumentales. Las primeras se refieren a la clara invasión en la privacidad de los deportistas que implicaba esta medida. Las segundas, a las consecuencias negativas que tendría para la reputación de la propia AMA la adopción de una medida tan drástica para asegurar el cumplimiento de las reglas de localización de los deportivas fuera de la competición. Es decir, que una vez que la evolución de la tecnología permita un uso respetuoso con los derechos de los deportistas al tiempo que efectivo en la lucha contra el dopaje no hay razón para oponerse a ello. De hecho, la AMA financia en la actualidad dos proyectos de investigación sobre el uso de la IA en la lucha contra el dopaje. El primero tiene como objetivo hacer más precisa la detección de EPO. El segundo tiene idéntica finalidad en relación con los esteroides.

\subsection{Prevención de lesiones.}

La prevención de lesiones es otra de las aplicaciones importantes de la IA. Esta función preventiva no solo beneficia al rendimiento y bienestar físico de los deportistas. Tiene también importantes consecuencias para el rendimiento en equipo y para evitar las pérdidas económicas que las lesiones suelen causar a los clubes deportivos. Generalmente estos sistemas se basan en algoritmos que predicen el riesgo de lesión y permiten gestionar de forma más eficiente los descansos y rotaciones entre jugadores. No en vano el desarrollo y comercialización de estos

\footnotetext{
${ }^{5}$ Geolocalisation of athletes for out-of-competition drug testing: ethical considerations. Position statement by the WADA Ethics Panel: https://bjsm.bmj.com/content/bjsports/early/2018/02/26/bjsports2017-098299.full.pdf
}

Citius, Altius, Fortius Volumen 13 ne 2. Noviembre, 2020. ISSH. 2340 9886.

http://doi.org/10.15366/eitius2020.13.2 
sistemas de prevención ha experimentado un notable auge. Normalmente estos equipos trabajan en tres etapas, a saber,

1. Recopilación de datos en el entrenamiento

2. Análisis del riesgo de lesión del atleta y del efecto acumulado

3. Recomendaciones a los entrenadores y resto del equipo técnico para prevenir las lesiones.

Un esquema ciertamente tan sencillo como inimaginable sin la existencia de este tipo de tecnología avanzada. Piénsese por ejemplo en la importancia que cobra estos sistemas en la prevención de lesiones en tiempos de la pandemia de COVID. La suspensión temporal de las competiciones obliga a los deportistas a modificar los entrenamientos y las rutinas de preparación física y psicológica. La vuelta a la competición después de estos períodos ha demostrado ser perjudicial para muchos deportistas. No cabe duda que el uso de esta tecnología en estas circunstancias permite detectar prematuramente el riesgo de lesiones derivadas de la falta de una adecuada la preparación física y psicológica.

Hay obviamente muchas otras aplicaciones de la IA al deporte, como la recopilación de datos en tiempo real de variables fisiológicas y otros indicadores que procesados adecuadamente por sistemas de aprendizaje automático permiten mejorar la nutrición de los atletas y aumentar la resistencia y el rendimiento deportivos. Por no mencionar siquiera la infinidad de usos que la IA tiene en los medios de comunicación especializados en el deporte, la promoción de los clubes o la interacción con los aficionados.

\section{El reto que supone la IA para los Derechos Humanos.}

Estas y otras aplicaciones de la IA en el deporte tienen también una vertiente menos atractiva. Muchas de las anteriores, sino todas, pueden ser utilizadas para manipular la competición y los deportistas. Nótese que la característica común de todas ellas es que reducen el ámbito de decisión de los seres humanos. Es decir, la autonomía de los deportistas, entrenadores, árbitros y resto de personas implicadas en el deporte. Aunque solo sea por esta razón es necesario reflexionar sobre las consecuencias éticas y jurídicas que el uso de la IA puede tener en el deporte. Porque si de algo podemos tener certeza es que estas tecnologías forman ya parte del deporte de competición y poco a poco se extenderán a otros ámbitos, como el recreacional e incluso el educativo.

No hay razón para oponerse a ello. Es necesario, eso sí, disponer de criterios claros que permitan delimitar los usos confiables de la IA, y destinarla, después, a la protección integral de los deportistas, la integridad de la competición y también de la buena gobernanza del deporte mundial. De ser así la IA y la tecnología avanzada permitirán un desarrollo hasta ahora inimaginable de las capacidades de los deportistas, o lo que es lo mismo, seguirán haciendo bueno el ya viejo adagio, Citius, Altius, Fortius, del que con tanto orgullo hace bandera el Olimpismo.

El reto no es menor. Asegurar un uso equitativo de estas tecnologías que sea a un tiempo respetuoso con la igualdad en la competición y en el acceso a los recursos competitivos, que mejore la sostenibilidad de los eventos deportivos y contribuya al desarrollo y bienestar humano, como requieren los Principios Fundamentales de la Carta Olímpica, no se antoja desde luego una meta fácil de alcanzar.

Es necesario por tanto ser conscientes de los beneficios y riesgos derivados del creciente uso de estas tecnologías en el deporte. Y poca duda cabe que los desafíos éticos que ponen ante nosotros no son menores. Repárese tan solo en el perjuicio que puede causar a la privacidad de los deportistas la existencia incontrolada de grandes bases de datos con información relevante sobre su biología y biografía. Y el perjuicio que esto puede suponer para su vida personal y profesional durante o después de finalizada la carrera deportiva. Tampoco son pocos los restos que la IA presenta a la gobernanza del deporte. Los sistemas de IA facilitan los procesos de toma de decisiones, pero pueden poner también en riesgo los procesos participativos y el compromiso 
de transparencia y responsabilidad que tímidamente ha comenzado a cobrar importancia en el seno de los organismos deportivos. Si a ello añadimos la creciente autonomía que adquieren estas tecnologías y la opacidad que presentan para nosotros es fácil percatarse de la necesidad de mantener el control sobre ellas antes de que su impacto en nuestras vidas y prácticas deportivas sea inmanejable.

\subsection{El compromiso con los Derechos Humanos como principio básico del uso de los sistemas de IA.}

El respeto de los Derechos Humanos es un compromiso moral y legal básico de la buena gobernanza del deporte. No es casualidad que la Carta Olímpica reconozca la práctica del deporte como un derecho humano que debe ser disfrutado sin discriminación de ningún tipo. ${ }^{6}$ De ahí que ante el riesgo que estas tecnologías pueden suponer para los derechos básicos de los deportistas y de otras personas involucradas en el deporte, sea importante asegurar que esos usos están dirigidos a respetar y reforzar al menos los siguientes derechos básicos ${ }^{7}$ :

I. Dignidad. La igual dignidad de todos los seres humanos fue definida hace ya tiempo por Kant como un imperativo categórico, o lo que es lo mismo, como una obligación moral ineludible. ${ }^{8}$ Como no podía ser de otra manera este es también el objetivo declarado del Olimpismo. Tal y como afirma el segundo Principio Fundamental de la Carta Olímpica, la finalidad del Movimiento Olímpico es «poner el deporte al servicio del desarrollo armonioso de la humanidad y promover una sociedad pacífica preocupada por la preservación de la dignidad humana». ${ }^{9}$ La protección de la dignidad tiene dos vertientes estrechamente vinculadas. Por una parte, en su sentido positivo o de acción, implica la obligación de adoptar medidas adecuadas para su protección por parte de los organismos que gobiernan el deporte. Por otra, en el sentido negativo o de omisión, exige la abstención de cualquier acción que pueda ponerla en riesgo o que suponga una intromisión ilegítima en la misma, como sucede siempre que se utiliza a los deportistas como medios para alcanzar un fin, aunque sea tan loable como la lucha contra el dopaje. De ahí que el uso de la IA en el deporte deba ser consecuente con el valor intrínseco de cada ser humano. No en vano, el riesgo de la generalización de la IA en el deporte es la consideración de los deportistas como meros objetos que pueden ser manipulados e instrumentalizaos sin reparos con la finalidad de obtener éxitos deportivos, aumentar los beneficios económicos de los clubes o, lo que es aún peor, ser utilizados como cobayas humanas para probar la eficiencia de ciertos dispositivos de tecnología avanzada que pueden llegar a anular completamente su dignidad y autonomía personales. En resumen, la dignidad humana como imperativo moral categórico impone la obligación de no instrumentalizar a los deportistas y otros interesados en el deporte.

II. Autonomía y libre desarrollo de la personalidad. Muy ligada a la dignidad personal, la autonomía es uno de los valores más preciados que poseemos los seres humanos. Ciertamente sin autonomía no es posible desarrollar los planes de vida individuales. Cualquier intrusión injustificada de otros individuos u órganos de gobierno del deporte pueden poner en peligro el libre desarrollo de la personalidad individual. Al igual que sucede con la dignidad, un compromiso serio con la autonomía individual implica un doble requisito. Por un lado, impone a las instituciones la obligación de proteger la autonomía individual con medidas adecuadas que aseguren y promuevan su ejercicio, y por otro abstenerse de ponerla en riesgo con medidas que coarten su disfrute. Esto es

\footnotetext{
${ }^{6}$ Olympic Charter. Fundamental Principles of Oympism. Principle 4. Available at https://stillmed.olympic.org/media/Document\%20Library/OlympicOrg/General/EN-OlympicCharter.pdf\#_ga=2.161010365.1213006632.1593117675-1057959592.1592862908

${ }^{7}$ High-Level Expert Group (2019), Ethic Guidelines for Trustworthy AI. Available at https://ec.europa.eu/digitalsingle-market/en/news/ethics-guidelines-trustworthy-ai

${ }^{8}$ Kant (1997, 4: 433-436)

${ }^{9}$ Véase el 2 Principio Fundamental de la Carta Olímpica.
} 
particularmente importante en el caso de los sistemas de inteligencia artificial utilizados para la vigilancia de los deportistas, muchas veces injustificada e injusta, o para manipularlos física y psicológicamente con consecuencias tan graves como la pérdida de la identidad personal. Ciertamente, la protección y promoción de la autonomía individual es particularmente importante para evitar las manipulaciones a las que son sometidos los deportistas por parte de entrenadores, médicos, psicólogos y por las propias organizaciones que gobiernan el deporte mundial. Este es ciertamente el riesgo que presentan las nuevas tecnologías inteligentes implantadas en el cuerpo de los deportistas que ya están siendo utilizados en el deporte.

III. Igualdad. La igualdad básica, ligada también con la igual dignidad personal, es un requisito político que justifica la existencia de instituciones sociales destinadas a establecer y promover los derechos básicos, como defendieron Hobbes ${ }^{10}$ y Hart ${ }^{11}$. De todas formas, probablemente haya sido Kant quien mejor expresó el alcance moral de la idea de la igualdad básica a través de la transición de los seres humanos como meros accidentes naturales (homo phaenomenon) a seres morales (homo noumenon) ${ }^{12}$ Es decir, el reconocimiento de la igual dignidad de todos los seres humanos, que impone, al mismo tiempo, el deber de respeto mutuo, como acabamos de ver en los casos de la dignidad y autonomía individuales. La consolidación de la idea de igualdad básica o igualdad profunda, como la llama Dworkin,$^{13}$ es según Scanlon, el progreso moral más importante de la humanidad. ${ }^{14}$ Aunque la igualdad es un concepto extremadamente vago y por ello difícil de evaluar es también muy importante en el deporte. No sólo porque el deporte sea un derecho humano cuyo disfrute no admite discriminación, sino porque la propia idea de fair play deportivo implica un fuerte compromiso con la igualdad de oportunidades. ${ }^{15}$ Este compromiso puede verse afectado también por los sistemas de IA. Ciertamente el desarrollo de los dispositivos tecnológicos y los sistemas de IA son extremadamente desiguales y pueden conducir a situaciones injustas y, lo que es peor, a evaluaciones sesgadas del principio de igualdad. Resulta por ello de vital importancia que la implementación de estos sistemas se lleve a cabo en consonancia con los principios de solidaridad, no discriminación y protección de los grupos vulnerables, como las mujeres, los deportistas de género no binario y los deportistas con discapacidad.

IV. Democracia. El compromiso con la democracia es otro principio básico de la buena gobernanza del deporte. La Carta Olímpica, el Código Ético y los Principios de buena Gobernanza del deporte promovidos por el COI imponen a todos las instituciones que conforman el Movimiento Olímpico la implementación de procesos democráticos de decisión y elecciones libres. Ahora bien, la democracia no debe ser entendida como un mero procedimiento formal de toma de decisiones. Más bien, este procedimiento formal es la última etapa de un proceso fuertemente comprometido con el resto de los requisitos anteriormente señalados. Es decir, lo que en la filosofía política se conoce como procesos democráticos que excluyen la dominación. ${ }^{16} \mathrm{El}$ concepto de democracia como no dominación requiere al menos que se cumplan las siguientes exigencias: a) el control efectivo del poder político de quienes se encuentran bajo el ámbito de decisión de los órganos de gobierno, b) la igualdad política entre ellos, o lo que es lo mismo, la igual posibilidad de hacer valer sus preferencias y, c) la promoción de procesos deliberativos en los que todas las partes puedan expresar libremente sus opiniones y tratar de influir en los demás a través de argumentos sustentados en razones públicas. No cabe duda de que los sistemas de IA pueden ayudar a mejorar y fortalecer los procesos democráticos. Entre

\footnotetext{
${ }^{10}$ Hobbes (1991 and 1993)

${ }^{11}$ Hart $(1994,193$ ss $)$

12 Kant (1997, 4: 433-436)

${ }^{13}$ Dworkin $(1985,273)$

${ }^{14}$ Scanlon $(2013,5)$

${ }^{15} \mathrm{He}$ desarrollado más a fondo sobre las implicaciones del concepto de igualdad en el deporte en Carrio (2021)

${ }^{16}$ Bessons \& Martí $(2018,7)$
} 
otras cosas porque proporcionan más seguridad en el control individual del voto y evitan manipulaciones de los resultados. Los sistemas de IA contribuyen de este modo a evitar el fraude y la corrupción en la elección de los cargos representativos que tantos males han causado al deporte. En la medida en que procesan eficazmente las preferencias individuales, estos sistemas pueden servir de ayuda en la toma de decisiones y aumentar así la participación en los asuntos del deporte. Pero también pueden resultar una grave amenaza para el proceso democrático. En primer lugar, porque facilitan el incremento de las decisiones automatizadas. En segundo lugar, y como consecuencia de lo anterior, puede alterar la igualdad de valor del voto individual al discriminar a aquellos que pudiendo hacerlo no participaron en proceso anteriores de decisión. En definitiva, estas nuevas tecnologías pueden erosionar seriamente la importancia de los procesos deliberativos y transformar por completo el procedimiento de decisión democrático.

\section{Algunos principios éticos que debe respetar el uso de la IA en el deporte.}

Aparte del compromiso mínimo con los Derechos Humanos al que me acabo de referir, es necesario disponer de un marco ético o moral que informe las posibles aplicaciones y desarrollo de la IA en el deporte. Es decir, un conjunto de principios que permitan asegurar un uso moralmente responsable de la IA y orientado a fortalecer los principios éticos que expresa la Carta Olímpica.

El compromiso moral de la IA con el bienestar de las personas y del planeta es también la principal preocupación que han mostrado los diferentes grupos de estudio de la IA en diversos ámbitos. Así ocurre, por mencionar tan solo algunos ejemplos bien conocidos con el Grupo Europeo sobre el Desarrollo Ético en la Ciencia y las Nuevas Tecnologías (EGE, por sus siglas en inglés), el Grupo de Expertos de Alto Nivel en Inteligencia Artificial (AIHLEG, por sus siglas en inglés), antes mencionados, y la Declaración de Montreal para un desarrollo responsable de la Inteligencia Artificial. ${ }^{17}$ Todos y cada uno de estos grupos han propuesto un conjunto de principios éticos básicos como un requisito mínimo para un uso seguro y responsable de la IA. Siguiendo estas recomendaciones y valiéndome además de los principios básicos de la bioética, tal como fueron los enunciados por Beauchamp y Childress (2008), propongo el siguiente conjunto de principios éticos como requisito mínimo e indispensable para una aplicación y desarrollo moralmente responsable de la IA en el deporte:
I. Principio de autonomía humana,
II. Principio de no maleficencia,
III. Principio de beneficencia
IV. Principio de justicia.
V. Principio de transparencia y responsabilidad.

En lo que sigue trataré de ofrecer una breve explicación de la importancia de cada uno de estos principios.

I. El principio de autonomía humana es un requisito ético fundamental que debe cumplir todo uso y aplicación de los sistemas de IA consecuente con el desarrollo humano. En otras palabras, es necesario asegurar que la IA no afecte negativamente a la autodeterminación de los seres humanos en el deporte. La importancia de este principio radica en que opera como criterio de orientación de las aplicaciones de la IA al deporte de tal modo que estas quedan subordinadas al bienestar y desarrollo humano, y no a la inversa, como ocurre no pocas veces al poner a los deportistas al servicio del desarrollo tecnológico. Así las cosas, toda aplicación de la IA en el deporte debe centrarse en incrementar las posibilidades creativas de los seres humanos y promover el deporte en todos sus ámbitos como un aspecto fundamental del bienestar y desarrollo de la humanidad. Por otra parte, el principio de autonomía humana debe

\footnotetext{
${ }^{17}$ https://www.montrealdeclaration-responsibleai.com/the-declaration 
ser también el que oriente la gobernanza del deporte y limite el uso de los procesos automatizados de decisión que den al traste con la incipiente democratización del deporte.

II. El principio de no maleficencia impone el deber ético de no causar daño a los deportistas ni ponerlos en situaciones de riesgo que puedan derivar en lesiones físicas y/o psicológicas. Este principio previene del uso negligente de los sistemas de IA e impone la responsabilidad de evitar el daño por acción u omisión en las diferentes aplicaciones de la IA en el deporte. Como es evidente la implementación de este principio debe regir la acción de entrenadores, equipos médicos, preparadores físicos y resulta particularmente relevante en el deporte infantil y juvenil, así como en el denominado deporte paralímpico y todas aquellas prácticas deportivas en las que se involucren deportistas con algún grado de discapacidad.

III. De forma similar a lo que ocurre con la no maleficencia, también el principio de beneficencia impone el deber de emplear los sistemas de IA en beneficio del desarrollo de los deportistas y del deporte en general como instrumento al servicio del desarrollo humano. Este principio, que podría ser visto como el reverso del principio de no maleficencia, suele entenderse, sin embargo, en un sentido más restringido. Es decir, mientras que el principio de maleficencia implica el deber general de no causar daño, el de beneficencia tan solo impondría la obligación, mucho más limitada, de actuar en beneficio de la persona. Probablemente esto sea cierto en muchos ámbitos relativos a la salud e integridad física y psíquica de los deportistas. Pero el principio de beneficencia puede entenderse también en un sentido más amplio. En particular si se relaciona con el concepto de igualdad básica y el principio de equidad como pilares básicos del deporte. En este sentido, el principio de beneficencia debe servir como criterio orientador de los usos de IA que deben emplearse en beneficio de los deportistas como seres humanos; también deben tener como finalidad la distribución equitativa del acceso a estos sistemas y vigilar porque sus aplicaciones se orienten a la sostenibilidad del deporte.

IV. El Principio de equidad es por sí mismo un compromiso del deporte que impone el deber de justicia en todos los ámbitos y obviamente también en las aplicaciones presentes y futuras de la IA. El problema es que la equidad y la justicia son conceptos que se prestan a interpretaciones diversas y, en ocasiones, incompatibles. Pero esto no es óbice para dejar de perseguir una distribución equitativa de estos recursos y evitar toda discriminación en el acceso a los beneficios que proporcionan por razones de las que los individuos no pueden hacerse responsables como el género, la raza o el lugar de nacimiento. Es decir, el principio de equidad debe orientarse al disfrute equitativo de las oportunidades y recursos disponibles en el deporte. Piénsese tan solo en la eficiencia de estos sistemas en la lucha contra el dopaje o en la mejora del rendimiento deportivo. No cabe duda de que una distribución desigual o la exclusión en su disfrute generan situaciones injustas y son susceptibles de degenerar en procesos de manipulación de los deportistas y de la competición. El principio de equidad tiene además una vertiente procesal relacionada con los principios de transparencia y responsabilidad que veremos a continuación.

V. Principio de transparencia y responsabilidad. La transparencia y la rendición de cuentas son dos principios básicos de la buena gobernanza del deporte promovida por el COI y de obligado cumplimiento para todas las organizaciones parte del Movimiento Olímpico. Pero el requisito de transparencia y responsabilidad resulta además fundamental en el caso de la IA por las siguientes razones. En primer lugar, la transparencia disminuye las sospechas de corrupción, desgraciadamente tantas veces confirmadas en el mundo del deporte. En segundo lugar, la transparencia incrementa la confianza en los órganos de gobierno la cual resulta fundamental en un tema jurídica y moralmente tan sensible como la IA. En tercer lugar, porque la transparencia en el uso y aplicaciones de estos sistemas en el deporte facilitan la exigencia de responsabilidad y reparación por los daños que pudieran producir. Por último, pero no menos importante, porque dada la creciente opacidad de estos sistemas cuanto más transparente sea su implementación más fiable y segura será su implantación en el deporte que debe quedar abierta a un debate científico y ético orientado a asegurar un uso seguro y 
confiable de estos sistemas. En otras palabras, el escrupuloso cumplimiento de estos requisitos es la mejor manera de asegurar la buena gobernanza en el deporte en general y de las aplicaciones presentes y futuras de la IA en particular. Nótese que estos requisitos son esenciales para seguir defendiendo con cierto grado de credibilidad la exigencia de autonomía que con tanto celo protegen los órganos de gobierno del deporte global.

\section{Conclusiones}

Como ocurre en todos los ámbitos de las creaciones humanas, la IA y la tecnología avanzada no son ni buenas ni malas en sí mismas. Su bondad o maldad, depende como dije al comienzo del uso que se haga de ellas. Dado que el deporte es un ámbito proclive a la experimentación y ha sido utilizado en no pocas ocasiones como campo de ensayo, es importante adelantarse a los posibles efectos adversos que estos sistemas de tecnología avanzada puedan tener.

No cabe duda de que un desarrollo confiable de la IA en el deporte puede comportar muchos beneficios. Tampoco de que su rápido despliegue ha modificado para siempre el mundo del deporte. Pero esto no es muy diferente de lo que ha ocurrido con la ropa y el calzado diseñado especialmente para el deporte, la constante mejora de la tecnología deportiva o, en fin, cualquier otra aplicación científica y tecnológica en el deporte. De ahí que como ocurre también en otros ámbitos de la vida no haya razón alguna para oponerse a un desarrollo controlado de la IA en el deporte. Al fin y al cabo, los algoritmos no entienden de ética y ninguno de estos sistemas e instrumentos son responsables de los usos que los humanos hacemos de ellos.

Pero no debemos olvidar que por primera vez en la historia de la humanidad estamos ante una situación en la que el control de estos sistemas puede quedar literalmente fuera de nuestras manos. De ahí que sea necesario e incluso urgente disponer de criterios éticos y de instrumentos jurídicos que permitan evaluar y regular los diferentes desarrollos de estos sistemas para adelantarse a los problemas que puedan generar. Por lo pronto es necesario retener al menos tres cuestiones básicas, a saber:

a) El riesgo de consolidar los sesgos padecidos en la creación de los algoritmos y las fuentes de información de los que se alimentan los sistemas de IA. Con el consiguiente peligro de que se incrementen exponencialmente los efectos perjudiciales que tienen en el deporte.

b) El riesgo de comenzar una carrera incontrolada en los usos y desarrollo de la IA en el deporte que puede llegar a tener consecuencias imprevisibles para la salud e integridad física de los deportistas y la sostenibilidad y autonomía del deporte.

c) La creciente opacidad de estos sistemas y el riesgo de que los seres humanos acabemos teniendo una posición subordinada ante ellos. ellos. Dado que el deporte seguirá siendo uno de los ámbitos más proclives al uso de estas tecnologías tratemos al menos de evitar que quede completamente dominado por ellas.

En definitiva, es mucho lo que hay en juego por lo que es preciso asegurar una estrategia confiable de la IA para que no acabe por dejarnos fuera de juego.

\section{Referencias bibliográficas.}

- Baca, A. (2012). Methods for Recognition and Classification of Human Motion Patterns. A Prerequisite for Intelligent Devices Assisting in Sports Activities. MATHMOD, 14, 15-17. Vienna, Austria. https://doi.org/10.3182/20120215-3-AT-3016.00009

- Besson, S. \& Martí, J.L. (2018). Legitimate actors of international law-making: towards a theory of international democratic representation. Jurisprudence. https://doi.org/10.1080/20403313.2018.1442256 
- Carrio Sampedro, A. (2021). La igualdad en el deporte. En Carrio, A., y Pérez Triviño, J.L., Introducción a la filosofía, ética y derecho del deporte. Mexico D.F., Fontamara. En prensa.

— Dworkin, R. (1985). A matter of principle. Cambridge, Mass: Harvard University Press.

- European Group On Ethics In Science And New Technologies (2018). Statment on Artificial Intelligence, Robotics and Autonomous systems. Available at https://ec.europa.eu/research/ege/pdf/ege_ai_statement_2018.pdf

— Hart, H.L.A. (1961). The Concept of Law. Oxford: Oxford University Press, 1994, 2 a ed.

- High-Level Expert Group (2019). Ethic Guidelines for Trustworthy AI. Available at https://ec.europa.eu/digital-single-market/en/news/ethics-guidelines-trustworthy-ai

- Hobbes, T. (1642). De Cive. En Rodríguez Feo, J. (ed.), El ciudadano. Madrid: Debate/CSIC, 1993.

- Hobbes, T. (1651). Leviathan. En Tuck, R. (Ed.), Hobbes: "Leviathan". Cambridge: Cambridge University Press, 1991.

- Kant, I., \& Korsgaard, C. (1998). Kant: Groundwork of the Metaphysics of Morals. En M. Gregor (Ed.), Cambridge Texts in the History of Philosophy. Cambridge: Cambridge University Press. https://doi.org/10.1017/CBO9780511809590

- McCullagh, J. (2010). Data Mining in Sport: A Neural Network Approach. International Journal of Sports Science and Engineering 4(3), 131-138.

- Montreal Declaration For A Responsible Development Of Artificial Intelligence, available at: https://www.montrealdeclaration-responsibleai.com/the-declaration

- Novatchkov, H., \& Baca, A. (2013), Artificial Intelligence in Sports on the Example of Weight Training. Journal of Sports Science and Medicine (12), 27-37.

— Scanlon, T.M. (2013). When Does Equality Matter? London: Oxford University Press. Disponible en línea: https://www.law.nyu.edu/sites/default/files/upload_documents/Lecture\%201\%20revised\%2 0October\%202014.pdf 